\title{
OUTLINE OF NGUETSENG'S APPROACH TO NON-PERIODIC HOMOGENIZATION
}

\author{
JAN FRANCÜ
}

\begin{abstract}
Classical homogenization theory developed for modeling heterogeneous media with regular fine structure is based on the assumption that the structure is periodic. Since the structure of real materials is not perfectly periodic, several approaches were proposed. The approach proposed by Gabriel Nguetseng in 2003 seems to be the most general deterministic approach, it covers periodic, almostperiodic and other structures. It is based on the notion of Banach algebra spectrum. The aim of this survey paper is to give a brief self-contained outline of this rather complicated approach described in many-page papers.
\end{abstract}

\section{INTRODUCTION}

Heterogeneous media with fine structure appear in many areas of human activities: in physics, chemistry, biology, earth sciences, economy, but mainly in technology. These composite materials with more or less periodic or even random structure are widely used in mechanical, civil and other engineering for its special properties.

Numerical computing of these materials by classical methods used for homogeneous materials is impossible due to the fine structure, which would require very fine triangulation in the finite element method and thus solving a very extensive system of equations. For computing reasons, this highly heterogeneous material must be modeled as a fictitious globally equivalent homogeneous material. What are the properties of this equivalent homogeneous material? In general, they differ from the usual weighted averages of properties of its components.

The mathematical method developed for this averaging is called homogenization. Following the approach introduced by I. Babuška in 1974, instead of one periodic material, the method considers a sequence of materials with refining structure "converging" to a fictitious homogeneous material. In mathematical setting, instead of one equation with periodic coefficient $a(x)$, the sequence of equations with coefficients $a^{\varepsilon}(x)=a\left(\frac{x}{\varepsilon}\right)$ with diminishing period $\varepsilon \rightarrow 0$ is studied. The limit $u^{*}$ of their solutions $u^{\varepsilon}$ is the solution to the so-called homogenized equation with homogenized constant coefficient $b$.

Many results were obtained for periodic structures and for many linear and some nonlinear equations, stationary and evolutionary problems: convergence of

$M S C$ (2010): primary 35B27; secondary 46J10.

Keywords: non-periodic homogenization, $\Sigma$-convergence, Banach algebra spectrum.

The author acknowledges a partial support from the Grant Agency of Brno University of Technology, project no. FSI-J-12-1817. 
the solutions, formulae for the homogenized coefficients. In addition, the so-called correctors added to the homogenized solution $u^{*}$ yield a better approximation of the solution $u^{\varepsilon}$ to the original periodic problem with $\varepsilon$-periodic coefficients, for details see [2].

Nevertheless the real materials are not exactly periodic, their structure is often completely random. The methods can be divided into two groups: in the first group the structure is supposed to be non-periodic but deterministic, the second one assumes a stochastic character of the structure, see, e.g. [12].

In the deterministic approach the assumption of periodic structure, i.e. assumption of periodic coefficients, was generalized, e.g., to non-uniformly periodic $a^{\varepsilon}(x)=a\left(x, \frac{x}{\varepsilon}\right)$ where $a(x, y)$ is periodic in the $y$ variable.

In 1989 G. Nguetseng invented in [8] two-scale convergence, which appeared to be a very efficient tool in periodic homogenization. In 2004 G. Nguetseng introduced a general approach to deterministic homogenization for non-periodic structure in a series of papers $[9,10,11]$. The assumption of periodicity of the structure is replaced by the existence of a spectrum of the so-called homogenization algebra and the two-scale convergence is replaced by a new $\Sigma$-convergence.

Since reading long many-page papers $[9,10,11]$ is not easy, the aim of this survey paper is to give the reader a self-contained introduction to studying this new abstract and rather difficult but powerful general approach to non-periodic homogenization. We start with a sketch of periodic homogenization with twoscale convergence, for more details see $[2,3,4,5]$. Then we specify basic tools for the general approach, in particular spectrum of a Banach algebra, for further details see $[6,14]$. Using Gelfand mapping between the homogenization algebra and the space of continuous functions on the spectrum, we can introduce a series of function spaces on the spectrum. After defining the $\Sigma$-convergence, application to homogenization is outlined. For details see $[9,10]$, for further results, we refer to $[13,7]$ and references therein.

\section{Periodic homogenization}

Before dealing with non-periodic homogenization, let us recall the formulation of the periodic homogenization problem. As a model problem we consider a boundary value problem for a second order elliptic partial differential equation on a bounded domain $\Omega$ in $\mathbb{R}^{N}$ with a "good" boundary $\partial \Omega$

$$
-\sum_{i, j=1}^{N} \frac{\partial}{\partial x_{i}}\left(a_{i j} \frac{\partial u}{\partial x_{j}}\right)=f \quad \text { in } \Omega, \quad u=0 \quad \text { on } \partial \Omega
$$

with matrix $\left\{a_{i j}\right\}_{i, j=1}^{N}$ of bounded coefficients $a_{i j}(x)$ satisfying the ellipticity condition with a constant $\alpha>0$

$$
\sum_{i, j=1}^{N} a_{i j}(x) \xi_{j} \xi_{i} \geq \alpha \sum_{i=1}^{N} \xi^{2} \quad \forall \xi \in \mathbb{R}^{N} \quad \forall x \in \Omega .
$$

Let $V=W_{0}^{1,2}(\Omega)$ be the Sobolev space of functions with zero traces on $\partial \Omega$ and square integrable derivatives. Then, for the coefficients $a_{i j}(x) \in L^{\infty}(\Omega)$ and for $f \in L^{2}(\Omega)$, the problem (2.1) admits a unique weak solution $u$, i.e., a function 
$u \in V$ satisfying

$$
\int_{\Omega} \sum_{i, j=1}^{N} a_{i j} \frac{\partial u}{\partial x_{j}} \frac{\partial v}{\partial x_{i}} \mathrm{~d} x=\int_{\Omega} f v \mathrm{~d} x \quad \forall v \in V .
$$

\section{Formulation of the homogenization problem}

Let $Y=\langle 0,1)^{N}$ be a unit cube in $\mathbb{R}^{N}$ serving as the basic period. Then we say that a function $a(y) \equiv a\left(y_{1}, \ldots, y_{N}\right)$ defined on $\mathbb{R}^{N}$ is $Y$-periodic if

$$
a\left(y_{1}, \ldots, y_{N}\right)=a\left(y_{1}+k_{1}, \ldots, y_{N}+k_{N}\right) \quad \forall y \in \mathbb{R}^{N} \forall k \in \mathbb{Z}^{N} .
$$

In the homogenization theory, sequences of problems are considered. A decreasing sequence $\left\{\varepsilon_{n}\right\}$ of small reals $\varepsilon_{n}$ tending to zero will be called scale. These $\varepsilon_{n}$ will label sequences, e.g. $a^{\varepsilon_{n}}, u^{\varepsilon_{n}}$, but the subscript $n$ in $\varepsilon_{n}$ will be omitted: $a^{\varepsilon}, u^{\varepsilon}$.

For a $Y$-periodic function $a$ and a scale $\{\varepsilon\}$, the relation

$$
a^{\varepsilon}(x)=a\left(\frac{x}{\varepsilon}\right) \equiv a\left(\frac{x_{1}}{\varepsilon}, \ldots, \frac{x_{N}}{\varepsilon}\right)
$$

defines a sequence of functions with diminishing period.

Let $\left\{a_{i j}\right\}$ be a matrix of $Y$-periodic $L^{\infty}$-functions satisfying ellipticity condition (2.2) and let $\{\varepsilon\}$ be a scale. Then (2.5) defines a sequence of coefficients $a_{i j}^{\varepsilon}(x)$. The homogenization problem consists in studying a sequence of boundary value problems (2.1) with coefficients $a_{i j}^{\varepsilon}$ with diminishing period $\varepsilon$ defined by (2.5)

$$
-\sum_{i, j=1}^{N} \frac{\partial}{\partial x_{i}}\left(a_{i j}^{\varepsilon} \frac{\partial u^{\varepsilon}}{\partial x_{j}}\right)=f \quad \text { in } \Omega, \quad u^{\varepsilon}=0 \quad \text { on } \partial \Omega
$$

and the behavior of the sequence of corresponding solutions $u^{\varepsilon}$.

In this model problem, the homogenization theory proves, see, e.g. [2], that:

- the sequence of solutions $u^{\varepsilon}$ converges (in $W^{1,2}(\Omega)$ weakly) to a function $u^{*}$,

- the limit $u^{*}$ is the so-called homogenized solution to the so-called homogenized problem, which is of the same form as (2.1) but with the so-called homogenized coefficients $b_{i j}$ :

$$
-\sum_{i, j=1}^{N} \frac{\partial}{\partial x_{i}}\left(b_{i j} \frac{\partial u^{*}}{\partial x_{j}}\right) \equiv-\sum_{i, j=1}^{N} b_{i j} \frac{\partial^{2} u^{*}}{\partial x_{j} \partial x_{i}}=f \quad \text { in } \Omega, \quad u^{*}=0 \quad \text { on } \partial \Omega
$$

being constant,

- the homogenized coefficients can be computed from the functions $a_{i j}$

$$
b_{i j}=\int_{Y}\left(a_{i j}-\sum_{k=1}^{N} a_{i k} \frac{\partial \chi^{j}}{\partial y_{k}}\right) \mathrm{d} y
$$

where $\chi^{j} \equiv \chi^{j}(y), j=1, \ldots, N$, are $Y$-periodic solutions to the equations

$$
-\sum_{i, k=1}^{N} \frac{\partial}{\partial y_{i}}\left(a_{i k} \frac{\partial \chi^{j}}{\partial y_{k}}\right)=-\sum_{i=1}^{N} \frac{\partial a_{i j}}{\partial y_{i}} \quad \text { on } Y
$$




\section{Crucial problem}

In the weak formulation of the so-called periodic problem: find $u^{\varepsilon} \in V$ such that

$$
\int_{\Omega} \sum_{i, j=1}^{N} a_{i j}^{\varepsilon} \frac{\partial u^{\varepsilon}}{\partial x_{j}} \frac{\partial v}{\partial x_{i}} \mathrm{~d} x=\int_{\Omega} f v \mathrm{~d} x \quad \forall v \in V,
$$

we have to pass to the limit as $\varepsilon \rightarrow 0$. The sequence of coefficients $a^{\varepsilon}$ is weakly converging. Due to the well known a priori estimate, the sequence of solutions $u^{\varepsilon}$ is bounded in $V$, thus containing a subsequence $u^{\varepsilon^{\prime}}$ weakly converging in $V$ and $\frac{\partial u^{\varepsilon^{\prime}}}{\partial x_{j}}$ weakly converge in $L^{2}(\Omega)$. This poses a problem. Since both sequences converge in $L^{2}(\Omega)$ only weakly, the limit of their product cannot be obtained from their weak limits as a simple counterexample shows:

Let $u^{\varepsilon}(x)=v^{\varepsilon}(x)=\sin \left(\frac{2 \pi x}{\varepsilon}\right)$. As $\varepsilon \rightarrow 0$, both sequences converge weakly to the zero function, but their product $u^{\varepsilon}(x) v^{\varepsilon}(x)=\sin ^{2}\left(\frac{2 \pi x}{\varepsilon}\right)=\frac{1}{2}\left[1-\cos \left(\frac{4 \pi x}{\varepsilon}\right)\right]$ converges to the $\frac{1}{2}$ constant function. Putting $v^{\varepsilon}(x)=\sin \left(\frac{2 \pi x}{\varepsilon}-\alpha\right)$ for different $\alpha$, the product $u^{\varepsilon} v^{\varepsilon}$ can converge to any constant function from the interval $\left\langle-\frac{1}{2}, \frac{1}{2}\right\rangle$.

This phenomenon is caused by the fact that the weak limit does not conserve information on the local behavior of the functions $u^{\varepsilon}$ and $v^{\varepsilon}$; in the weak limit, this local behavior is lost.

The problem was first overcome in 1970's by a special choice of oscillating test functions $v^{\varepsilon}$, see, e.g. [2], and later also by the so-called "div-curl lemma" by F. Murat and L. Tartar, which does not require periodicity assumptions.

\section{Two-scale convergence}

With a periodic structure, the problem of passing to the limit in the product of two weakly converging sequences can be solved by a limit of special "two-scale convergence" invented by Nguetseng in [8] and further developed by Allaire [1]. For a sequence of functions $u^{\varepsilon}$ of one variable $x \in \Omega$, the two-scale limit $u^{0}$ has two variables: variable $x \in \Omega$ describing the global behavior and variable $y \in Y$ containing the local behavior. Then, for $p \in\langle 1, \infty\rangle$ and a scale $\{\varepsilon\}$, we introduce:

Definition 2.1. A sequence $\left\{u^{\varepsilon}(x)\right\}$ in $L^{p}(\Omega)$ is said to converge two-scale (weakly) in $L^{p}(\Omega)$ to some $u^{0}(x, y) \in L^{p}(\Omega \times Y)$ as $\varepsilon \rightarrow 0$, if the sequence $\left\{u^{\varepsilon}\right\}$ is bounded in $L^{p}(\Omega)$ and the following convergence

$$
\int_{\Omega} u^{\varepsilon}(x) \varphi\left(x, \frac{x}{\varepsilon}\right) \mathrm{d} x \rightarrow \int_{\Omega}\left(\int_{Y} u^{0}(x, y) \varphi(x, y) \mathrm{d} y\right) \mathrm{d} x
$$

holds for all test functions $\varphi$ continuous on $\bar{\Omega} \times \bar{Y}$ and $Y$-periodic in $y$.

Next, the sequence $\left\{u^{\varepsilon}\right\}$ is said to converge two-scale strongly to some $u_{0}$ if, in addition, the corresponding norms converge

$$
\left\|u^{\varepsilon}\right\|_{L^{p}(\Omega)} \rightarrow\left\|u^{0}\right\|_{L^{p}(\Omega \times Y)} .
$$

Let us remark that the strong two-scale convergence implies the weak two-scale convergence and, if $p<\infty$, it implies the weak convergence in $L^{p}(\Omega)$. But in the case of $p=\infty$, the two-scale convergence implies only weak-* convergence in $L^{\infty}(\Omega)$. 
In the previous example with $Y=\langle 0,1)$, both sequences $u^{\varepsilon}(x)=\sin \left(\frac{2 \pi x}{\varepsilon}\right)$ and $v^{\varepsilon}(x)=\sin \left(\frac{2 \pi x}{\varepsilon}-\alpha\right)$ converge two-scale strongly to their two-scale limits $u^{0}(x, y)=\sin (2 \pi y), v^{0}(x, y)=\sin (2 \pi y-\alpha)$, respectively. Their product $u^{\varepsilon} v^{\varepsilon}$ converges two-scale strongly to $u^{0} v^{0}$ and weakly in $L^{2}(Y)$ to the function

$$
\int_{Y} u^{0}(x, y) v^{0}(x, y) \mathrm{d} y=\int_{0}^{1} \sin (2 \pi y) \sin (2 \pi y-\alpha) \mathrm{d} y .
$$

Let us introduce another example. Let $a \in L^{\infty}\left(\mathbb{R}^{N}\right)$ be a $Y$-periodic function with $\int_{Y} a(y) \mathrm{d} y=0$ and $f, g \in L^{2}(\Omega)$. Then the sequence

$$
u^{\varepsilon}(x)=f(x) a\left(\frac{x}{\varepsilon}\right)+g(x)
$$

converges weakly to $g(x)$ and two-scale strongly to $u_{0}(x, y)=f(x) a(y)+g(x)$.

But if the periods of $a^{\varepsilon}$ are not diminishing at the same velocity as the scale $\varepsilon$, i.e., if they are not "in resonance", e.g. $u^{\varepsilon}(x)=f(x) a\left(\frac{x}{\varepsilon \sqrt{2}}\right)+g(x)$, then $u^{\varepsilon}$ converges only two-scale weakly to the limit $g$, i.e., the information on the local behavior of $a^{\varepsilon}$ is lost as in the case of the weak $L^{2}(\Omega)$-limit.

Let us mention two important results on the two-scale convergence, which enables us to pass to the limit in (2.10):

Theorem 2.1. (Compactness) Let $u^{\varepsilon}$ be a sequence bounded in $L^{p}(\Omega)$. Then there exists a subscale $\left\{\varepsilon^{\prime}\right\} \subset\{\varepsilon\}$ and a limit $a^{0} \in L^{p}(\Omega \times Y)$ such that the subsequence $\left\{u^{\varepsilon^{\prime}}\right\}$ weakly two-scale converges to the limit $u^{0}$.

Theorem 2.2. (Limit PROCEDURE) Let the exponents $p, q, r \in\langle 1, \infty\rangle$ satisfy $\frac{1}{p}+\frac{1}{q}=\frac{1}{r}$ and let $u^{\varepsilon}$ strongly two-scale converge to $u^{0}$ in $L^{p}(\Omega)$ and $v^{\varepsilon}$ weakly two-scale converge to $v^{0}$ in $L^{q}(\Omega)$.

Then the products $u^{\varepsilon} v^{\varepsilon}$ weakly two-scale converge to the limit $u^{0} v^{0}$ in $L^{r}(\Omega)$.

In the model problem (2.6), the coefficients $a_{i j}^{\varepsilon}$ converge two-scale strongly in $L^{\infty}(\Omega)$ and the derivatives $\frac{\partial u}{\partial x_{i}}$ of the solution contain a subsequence converging two-scale weakly in $L^{2}(\Omega)$, thus we can pass to the limit.

For more details and further results on two-scale convergence including the unfolding approach, the papers $[3,4,5]$ can be recommended.

\section{NON-PERIODIC HOMOGENIZATION - BASIC TOOLS}

The general approach to non-periodic homogenization introduced by Nguetseng requires the introduction of several notions. Sequences will still be labeled by the scale $\{\varepsilon\}$, a decreasing sequence of positive $\varepsilon$ tending to zero.

The scale $\{\varepsilon\}$ with a mapping $H^{\varepsilon}: \mathbb{R}^{N} \rightarrow \mathbb{R}^{N}$ called scaling assigns to a function $a$ on $\mathbb{R}^{N}$ a sequence of functions $\left\{a^{\varepsilon}\right\}$ defined by $a^{\varepsilon}(x)=a\left(H^{\varepsilon}(x)\right)$. For the sake of simplicity, as in the periodic homogenization, we use the scaling $H^{\varepsilon}: x \mapsto \frac{x}{\varepsilon}$ for introducing the sequence of coefficients $a^{\varepsilon}$ for the sequence of equations.

Let us remark that a more general scaling $H^{\varepsilon}: \mathbb{R}^{N} \rightarrow \mathbb{R}^{N}$ can be used, e.g.,

$$
H^{\varepsilon}:\left(x_{1}, \ldots, x_{N}\right) \mapsto\left(\frac{x_{1}}{\varepsilon^{\alpha_{1}}}, \ldots, \frac{x_{N}}{\varepsilon^{\alpha_{N}}}\right)
$$


with parameters $\alpha_{i}>0$. The scaling should be a one parameter semigroup $\left\{H^{\varepsilon}\right\}_{\varepsilon>0}$ such that $\left|H^{\varepsilon}(x)\right| \rightarrow \infty$ as $\varepsilon \rightarrow 0$ for each $x \neq 0$ satisfying a measure transform law with a convenient function $m(\varepsilon)$, i.e. $\left|H^{\varepsilon}(M)\right|=m(\varepsilon)|M|$ for all bounded measurable subsets $M$ in $\mathbb{R}^{N}$.

\section{Basic function space $\mathcal{B C}\left(\mathbb{R}^{N}\right)$}

The basic function space will be $\mathcal{B C}\left(\mathbb{R}^{N}\right)$ - the space of all real functions bounded and continuous on $\mathbb{R}^{N}$. It is a real linear space. The pointwise product defined by $(u v)(x)=u(x) v(x)$ of bounded and continuous functions is also bounded and continuous. Thus, with this multiplication, the space $\mathcal{B C}\left(\mathbb{R}^{N}\right)$ is also an algebra, i.e., the linear space with an operation of multiplication that is

- associative: $(u v) w=u(v w)$,

- distributive: $u(v+w)=u v+u w$ and $(u+v) w=u w+v w$

- and satisfies: $\alpha(u v)=(\alpha u) v=u(\alpha v) \forall \alpha \in \mathbb{R}$.

The space $\mathcal{B C}\left(\mathbb{R}^{N}\right)$ contains an element $e$ satisfying $u e=e u=u$ called identity, in our case it is the constant function $e(x)=1$. Further, the multiplication is commutative, i.e., $v u=u v$. Thus, the space is called a commutative algebra.

Endowed with the supremum norm $\|u\|=\sup _{x \in \mathbb{R}_{N}}|u(x)|$, the linear space $\mathcal{B C}\left(\mathbb{R}^{N}\right)$ is a real complete normed linear space, i.e., Banach space. Since the multiplication satisfies $\|u v\| \leq\|u\| \cdot\|v\|$, it is called Banach algebra with the norm of the identity being $\|e\|=1$.

To sum up: the space $\mathcal{B C}\left(\mathbb{R}^{N}\right)$ with pointwise multiplication and supreme norm is a real commutative Banach algebra with identity.

Starting with the complex valued functions, we obtain the complex Banach algebra. More information on complex algebras can be found in $[6,14]$. In $[9$, $10,11]$ the algebra $\mathcal{B C}\left(\mathbb{R}^{N}\right)$ is assumed to be complex valued, for homogenization problems, the algebra of real valued functions is sufficient.

\section{Mean value on $\mathbb{R}^{N}$ and the space $\Pi^{\infty}\left(\mathbb{R}^{N}\right)$}

A function $u \in \mathcal{B C}\left(\mathbb{R}^{N}\right)$ has a mean value on $\mathbb{R}^{N}$ under scaling $H^{\varepsilon}: x \mapsto \frac{x}{\varepsilon}$, if there exists a constant $M(u) \in \mathbb{R}$ that is the weak ${ }^{*}$ limit of $u^{\varepsilon}(x)=u\left(\frac{x}{\varepsilon}\right)$ in $L^{\infty}\left(\mathbb{R}^{N}\right)$, i.e., for each $\varphi \in \mathcal{C}_{0}^{\infty}\left(\mathbb{R}^{N}\right)$,

$$
\int_{\mathbb{R}^{N}} u\left(\frac{x}{\varepsilon}\right) \varphi(x) \mathrm{d} x \rightarrow M(u) \int_{\mathbb{R}^{N}} \varphi(x) \mathrm{d} x \quad \text { as } \varepsilon \rightarrow 0 .
$$

The constant $M(u)$ is called the mean value of the function $u$. The space of real bounded continuous functions having mean values will be denoted by $\Pi^{\infty}\left(\mathbb{R}^{N}\right)$. It is a closed subalgebra of $\mathcal{B C}\left(\mathbb{R}^{N}\right)$ and $M$ is a positive linear form on $\Pi^{\infty}\left(\mathbb{R}^{N}\right)$.

\section{Homogenization algebra}

Nguetseng in [9] introduced some new concepts. Structural representation $\Gamma$ is a countable multiplicative group of non-negative functions in $\Pi^{\infty}\left(\mathbb{R}^{N}\right)$. It will be a basic set for coefficients modeling the structure of the material to be homogenized. Homogenization structure $\Sigma$ is a class of structural representations generating the same linear subspace in $\Pi^{\infty}\left(\mathbb{R}^{N}\right)$. 
The smallest complete subalgebra $A$ in $\Pi^{\infty}\left(\mathbb{R}^{N}\right)$ containing the constant functions and the structural representation $\Gamma$ is called a homogenization algebra or briefly $H$-algebra. Thus the $H$-algebra $A$ is a complete subalgebra of $\Pi^{\infty}\left(\mathbb{R}^{N}\right)$ containing constant functions. It is separable since $\Gamma$ is countable. The mean value operator $M$ is defined on $A$.

In the periodic case, the algebra $\mathcal{P}(Y)$ of all continuous $Y$-periodic functions (with fixed period $Y$ ) is a separable subalgebra of $\Pi^{\infty}\left(\mathbb{R}^{N}\right)$ containing constants, and thus, can serve as an $H$-algebra.

Beyond the periodic case is the algebra of all almost periodic function $\mathcal{A P}\left(\mathbb{R}^{N}\right)$. A function $u \in \Pi^{\infty}\left(\mathbb{R}^{N}\right)$ is called almost periodic if the set of all its translates

$$
\left\{\tau_{a} u \mid \tau_{a} u(x)=u(x-a), a \in \mathbb{R}^{N}\right\}
$$

forms a relatively compact set in $\Pi^{\infty}\left(\mathbb{R}^{N}\right)$. But the space $\mathcal{A P}\left(\mathbb{R}^{N}\right)$ is not separable. $H$-algebra can be obtained in the following way: we choose a countable collection of positive almost periodic functions and the smallest subalgebra containing these functions will be a separable subalgebra in $\Pi^{\infty}\left(\mathbb{R}^{N}\right)$, which is an $H$-algebra. For details see [11].

\section{Banach algebra spectrum}

This is the most important concept of Nguetseng's approach. Papers [9, 10, 11] simply say: Let $\Delta(A)$ be a spectrum of algebra $A$. For the reader not familiar with the theory of Banach algebras, let us add a brief explanation.

The well known notion of the spectrum $\sigma(L)$ of a linear mapping $L: X \rightarrow X$ is a set of all complex numbers $\lambda$ such that the mapping $L_{\lambda}: x \mapsto L x-\lambda x$ is not invertible, or the inverse $L_{\lambda}^{-1}$ is not continuous.

The spectrum of a Banach algebra is a different concept. By one of the definitions, the spectrum $\Delta(A)$ of a Banach algebra $A$ is the set of all nontrivial multiplicative linear forms on $A$.

Thus $\Delta(A)$ is a subset of the topological dual space $A^{*}$. Let us recall that a linear form $s: A \rightarrow \mathbb{R}$ conserves linear space operations $s(u+v)=s(u)+s(v)$ and $s(\alpha u)=\alpha s(u)$. Its continuity means that it conserves the norm convergence: $u_{n} \rightarrow u$ implies $s\left(u_{n}\right) \rightarrow s(u)$, which is equivalent to boundedness: $|s(u)| \leq c\|u\|$ where the smallest constant $c$ is the norm $\|s\|_{*}$ of the form $s$. The set of all continuous linear forms on $A$ with norm $\|\cdot\|_{*}$ is the so-called dual space $A^{*}$ which is also a Banach space.

The continuous linear form $s$ is called multiplicative if it moreover satisfies

$$
s(u v)=s(u) s(v) .
$$

The equality implies that the form $s$ is positive, i.e., $u>0 \Rightarrow s(u)>0$. Indeed, for $v$ defined by $v(x)=\sqrt{u(x)}>0$, we have

$$
s(u)=s(v v)=s(v) s(v)=(s(v))^{2}>0 .
$$

Equality (3.1) also implies that the norms of nontrivial multiplicative forms are $\|s\|_{*}=1$. Indeed, let $u>0$ be such that $s(u)=\|s\|_{*}\|u\|$ and again $v=\sqrt{u}$. Then

$$
\|s\|_{*}\|u\|=s(u)=s(v v)=s(v) s(v) \leq\|s\|_{*}^{2}\|v\|^{2}=\|s\|_{*}^{2}\left\|v^{2}\right\|=\|s\|_{*}^{2}\|u\|,
$$


which implies $\|s\|_{*} \geq 1$. The opposite inequality $\|s\|_{*} \leq 1$ can be obtained similarly from $\|s\|_{*}\left\|u^{2}\right\| \geq s\left(u^{2}\right)=s(u)^{2}=\|s\|_{*}^{2}\|u\|^{2}$.

Thus, the spectrum $\Delta(A)$ is not a linear subspace. Moreover, if $s \in \Delta(A)$, then no other multiple $\alpha s(\alpha \neq 1)$ is in $\Delta(A)$. Also, if $s_{1}, s_{2} \in \Delta(A)$, then $s_{1}+s_{2} \notin \Delta(A)$.

Dealing with a subalgebra of $\mathcal{B C}\left(\mathbb{R}^{N}\right)$, we prove another important properties. Let functions of algebra $A$ have independent values in different $x_{1}, x_{2} \in \mathbb{R}^{N}$, i.e., let $A$ contain functions $u_{1}, u_{2}$ satisfying $u_{1}\left(x_{1}\right)=u_{2}\left(x_{2}\right)=1$ and $u_{1}(x) u_{2}(x)=0$ for all $x \in \mathbb{R}^{N}$. Then

$$
s\left(u_{1}\right) s\left(u_{2}\right)=s\left(u_{1} u_{2}\right)=s(0)=0,
$$

which implies that either $s\left(u_{1}\right)=0$ or $s\left(u_{2}\right)=0$. This indicates that each form $s \in \Delta(A)$ can depend on values of $u$ at one "independent" point $x \in \mathbb{R}^{N}$ only. There is a one-to-one correspondence between the spectrum $\Delta(A)$ and "independent" subsets of $\mathbb{R}^{N}$. The algebra $A$ thus splits points of $\mathbb{R}^{N}$ into classes of independent points represented by multiplicative forms $s$ of the spectrum $\Delta(A)$.

For example, in the case of the algebra of $Y$-periodic functions on $\mathbb{R}^{N}$, the forms of the spectrum $\Delta(A)$ can be identified with points of the unit period $Y$ since the $Y$-periodic functions have the same value at corresponding points in all periods $Y+k$, i, e. $u(x+k)=u(x)$ holds for each $k \in \mathbb{Z}^{N}, y \in \mathbb{R}^{N}$. Thus, each form $s$ of the spectrum $\Delta(A)$ corresponds to a collection of points in $\mathbb{R}^{N}$.

The algebraic approach, see $[6,14]$, says that the spectrum $\Delta(A)$ can be identified with the set of all maximal ideals $\mathcal{I}_{x}$ in $A$. Each set $\mathcal{I}_{x}=\{u \in A \mid u(x)=0\}$ for $x \in \mathbb{R}^{N}$ is a maximal ideal. Indeed, since $u(x) v(x)=0$ for any $u \in \mathcal{I}_{x}$ and $v \in A$, the condition $\mathcal{I}_{x} A=A \mathcal{I}_{x}=\mathcal{I}_{x}$ is satisfied and $\mathcal{I}_{x}$ is an ideal. It is maximal since there is no ideal between $\mathcal{I}_{x}$ and $A$.

\section{Gelfand representation}

The Gelfand representation $\mathcal{G}$ maps the algebra $A$ onto the space $\mathcal{C}(\Delta(A))$ of continuous functions on the spectrum $\Delta(A)$ :

For a function $u \in A$, the image $\mathcal{G}(u)$ is a function on the spectrum $\Delta(A)$

$$
\mathcal{G}: u \in A \mapsto \mathcal{G}(u) \in \mathcal{C}(\Delta(A)) \quad \text { where } \quad \mathcal{G}(u)(s)=s(u) \quad \forall s \in \Delta(A) .
$$

The Gelfand representation conserves the norm: $\|\mathcal{G}(u)\|_{\mathcal{C}(\Delta)}=\|u\|_{A}$. Thus, the mapping $\mathcal{G}$ is an isometric isomorphism between $A$ and $\mathcal{C}(\Delta(A))$, i.e., a bijection conserving the operations and the corresponding norms.

\section{SpaCes on the SPeCtrum AND $\Sigma$-CONVERGENCE}

Using the mean value operator $M$ and the Gelfand mapping $\mathcal{G}$, we shall construct integral and differential structures on $\Delta(A)$ corresponding to those on $\mathbb{R}^{N}$.

\section{Induced measure and integration on the spectrum}

A mean value operator $M: \Pi^{\infty}\left(\mathbb{R}^{N}\right) \rightarrow \mathbb{R}$ induces a measure $\beta$ on the spectrum $\Delta(A)$. Since $\varphi \in \mathcal{C}(\Delta(A)) \mapsto M\left(\mathcal{G}^{-1}(\varphi)\right)$ is a continuous linear form on $\mathcal{C}(\Delta(A))$,

$$
M(u)=\int_{\Delta(A)} \mathcal{G}(u)(s) \mathrm{d} \beta(s)
$$


defines a Radon measure $\beta$ on the spectrum $\Delta(A)$. The measure $\beta$ thus expresses a measure of the corresponding set of "dependent points" in $\mathbb{R}^{N}$.

In the case of the algebra $A$ of $Y$-periodic continuous functions, $\Delta(A)$ can be identified with the period $Y$ and the measure $\beta$ is the Lebesgue measure on $Y$. The mean value operator $M$ can be written as $M(u)=\int_{Y} u(y) \mathrm{d} y$.

\section{Lebesgue spaces on the spectrum}

First, let $\Xi_{p} \quad(1 \leq p<\infty)$ be a space of all $u \in L_{l o c}^{p}\left(\mathbb{R}^{N}\right)$ such that

$$
\|u\|_{\Xi p} \equiv \sup _{0<\varepsilon \leq 1}\left(\int_{\{|x|<1\}}\left|u\left(\frac{x}{\varepsilon}\right)\right|^{p} \mathrm{~d} x\right)^{1 / p}<\infty .
$$

The space $\Xi_{p}$ with $\|\cdot\|_{\Xi_{p}}$ is a Banach space. Let $\mathcal{X}_{A}^{p}$ be the closure of the algebra $A$ in the space $\Xi_{p}$. The set $\mathcal{X}_{A}^{p}$ with the norm $\|\cdot\|_{\Xi_{p}}$ is also a Banach space.

Due to equality (4.1), the Gelfand representation $\mathcal{G}$ can be extended by continuity from $A$ to $\mathcal{X}_{A}^{p}$ to the mapping denoted again by $\mathcal{G}$. The image of $\mathcal{X}_{A}^{p}$ in $\mathcal{G}$ defines a Lebesgue space $L^{p}(\Delta(A))$ equipped with the norm

$$
\|\psi\|_{L^{p}(\Delta(A))}=\left[\int_{\Delta(A)}|\psi(s)|^{p} \mathrm{~d} \beta(s)\right]^{1 / p} \equiv\left[M\left(\mathcal{G}^{-1}\left(|\psi|^{p}\right)\right)\right]^{1 / p} .
$$

\section{Differentiation on $\mathcal{C}(\Delta(A))$ and further spaces}

Gelfand representation $\mathcal{G}$ makes it possible to differentiate functions on the spectrum $\Delta(A)$. The derivative $\frac{\partial \psi}{\partial s_{i}}$ of $\psi \in \mathcal{C}(\Delta(A))$ is constructed from the derivative of the corresponding function on $\mathbb{R}^{N}$ in the following way

$$
\frac{\partial \psi}{\partial s_{i}}=\mathcal{G}\left(\frac{\partial}{\partial x_{i}}\left(\mathcal{G}^{-1}(\psi)\right)\right) .
$$

It is defined for such $\psi$ that $\mathcal{G}^{-1}(\psi) \in A^{1}$ where

$$
A^{1}=\left\{u \in A \mid \frac{\partial u}{\partial x_{1}}, \ldots, \frac{\partial u}{\partial x_{N}} \in A\right\} .
$$

The space $\mathcal{D}^{1}(\Delta(A))$ is the image of the space $A^{1}$ in the mapping $\mathcal{G}$. The norm on $A^{1}$ induces a norm on $\mathcal{D}^{1}(\Delta(A))$. Analogously, higher order derivatives of functions on $\Delta(A)$ are introduced. In the same way, also subspaces $A^{k}, k=1,2,3, \ldots$ and $A^{\infty}$ of $A$ and by the Gelfand mapping the corresponding subspaces $\mathcal{D}^{k}(\Delta(A))$, $\mathcal{D}^{\infty}(\Delta(A))$ of $\mathcal{C}(\Delta(A))$ are defined.

We shall assume that $A^{\infty}$ is dense in $A$, which implies that $\mathcal{D}^{\infty}(\Delta(A))$ is dense in $\mathcal{C}(\Delta(A))$. Similarly, Sobolev type spaces on $\Delta(A)$ can be introduced. The space $W^{1, p}(\Delta(A))$ is defined as the closure of $\mathcal{D}^{1}(\Delta(a))$ in the norm

$$
\|\psi\|_{W^{1, p}(\Delta(A))}=\left[\|\psi\|_{L^{p}(\Delta(A))}^{p}+\sum_{i=1}^{N}\left\|\frac{\partial \psi}{\partial s_{i}}\right\|_{L^{p}(\Delta(A))}^{p}\right]^{1 / p} .
$$

For $p=2$, the space $W^{1,2}(\Delta(A)) \equiv H^{1}(\Delta(A))$ is a Hilbert space. The space $H^{1}(\Delta(A))$ factorized by $\mathbb{R}$ is denoted by $H^{1}(\Delta(A)) / \mathbb{R}$. 
Spaces on $\Omega \times \Delta(A)$

Let $\Omega$ be a bounded domain in $\mathbb{R}^{N}$ with a Lipschitz boundary $\partial \Omega$. Gelfand mapping $\mathcal{G}$ makes it possible to convert some functions defined on $\mathbb{R}^{N}$ into functions on $\Delta(A)$. We extend this $\mathcal{G}$ to mapping $\mathcal{G}_{\Omega}$ converting function $u \equiv u(x, y)$ on $\Omega \times \mathbb{R}^{N}$ into function $\widehat{u}(x, s)$ on $\Omega \times \Delta(A)$. Thus, $\mathcal{G}_{\Omega}$ is a continuous linear mapping between spaces $L^{p}\left(\Omega ; \mathcal{X}_{A}^{p}\right) \rightarrow L^{p}(\Omega \times \Delta(A))$ or $\mathcal{C}(\bar{\Omega} ; A) \rightarrow \mathcal{C}(\bar{\Omega} \times \Delta(A))$, etc.

Further, we shall also need functions of the type $u\left(x, \frac{x}{\varepsilon}\right)$, i.e., traces, which need some continuity. The simplest way is to take functions continuous on $\bar{\Omega} \times \Delta(A)$.

\section{$\Sigma$-convergence}

In the non-periodic homogenization, the role of two-scale convergence is played by $\Sigma$-convergence. Let $A$ be the $H$-algebra generated by a $H$-structure $\Sigma$.

Definition 4.1. The sequence $\left\{u^{\varepsilon}\right\}$ in $L^{p}(\Omega) \quad(1 \leq p \leq \infty)$ is said to be weakly $\Sigma$-convergent to some $u^{0} \in L^{p}(\Omega \times \Delta(A))$ if it is bounded in $L^{p}(\Omega)$ and

$$
\int_{\Omega} u^{\varepsilon}(x) v^{\varepsilon}(x) \mathrm{d} x \rightarrow \iint_{\Omega \times \Delta(A)} u_{0}(x, s) \widehat{v}(x, s) \mathrm{d} x \mathrm{~d} \beta(s)
$$

for each $v \in \mathcal{C}(\bar{\Omega}, A)$ where $v^{\varepsilon}(x)=v\left(x, \frac{x}{\varepsilon}\right)$ and $\widehat{v}=\mathcal{G}_{\Omega}(v)$.

Further, the sequence $\left\{u^{\varepsilon}\right\}$ in $L^{p}(\Omega)$ is said to be strongly $\Sigma$-convergent to some $u^{0} \in L^{p}(\Omega, \Delta(A))$ if $u^{\varepsilon}$ is weakly $\Sigma$-convergent to $u^{0}$ and

$$
\left\|u^{\varepsilon}\right\|_{L^{p}(\Omega)} \rightarrow\left\|u^{0}\right\|_{L^{p}(\Omega \times \Delta(A))} .
$$

The $\Sigma$-convergence has the same compactness and convergence property as the two-scale convergence:

Theorem 4.1. Each sequence $\left\{u^{\varepsilon}\right\}$ bounded in $L^{p}(\Omega)$ contains a subsequence $\left\{u^{\varepsilon^{\prime}}\right\}$ weakly $\Sigma$-converging to a limit $u^{0} \in L^{p}(\Omega \times \Delta(A))$.

Theorem 4.2. Let $\left\{u^{\varepsilon}\right\}$ be a sequence in $L^{p}(\Omega)$ and $\left\{v^{\varepsilon}\right\}$ in $L^{q}(\Omega)$ where $\frac{1}{p}+\frac{1}{q}=\frac{1}{r} \leq 1$. If $\left\{u^{\varepsilon}\right\}$ weakly $\Sigma$-converges to $u^{0} \in L^{p}(\Omega \times \Delta(A))$ and $\left\{v^{\varepsilon}\right\}$ strongly $\Sigma$-converges to $v^{0} \in L^{q}(\Omega \times \Delta(A))$, then the product $\left\{u^{\varepsilon} v^{\varepsilon}\right\}$ weakly $\Sigma$ converges to $u^{0} v^{0} \in L^{r}(\Omega \times \Delta(A))$.

\section{Application to the homogenization problem}

Let us outline how the method can be applied to the homogenization problem. We shall consider a simple boundary value problem similar to (2.6)

$$
-\sum_{i, j=1}^{N} \frac{\partial}{\partial x_{i}}\left(a_{i j}^{\varepsilon}(x) \frac{\partial u^{\varepsilon}}{\partial x_{j}}\right)=f \text { in } \Omega, \quad u^{\varepsilon}=0 \text { on } \partial \Omega .
$$

The coefficients $a_{i j}^{\varepsilon}$ are given by $a_{i j}^{\varepsilon}(x)=a_{i j}\left(\frac{x}{\varepsilon}\right)$ where $a_{i j} \in L^{\infty}\left(\mathbb{R}^{N}\right)$ and satisfy the ellipticity condition (2.2). Using standard methods, one can prove that, for each $\varepsilon>0$, the problem admits a unique solution $u^{\varepsilon} \in H_{0}^{1}(\Omega)$. The solution is bounded in the norm of $H_{0}^{1}(\Omega)$ by a constant independent of $\varepsilon$. 


\section{$H$-structure and $H$-algebra}

The coefficients $a_{i j}$ are supposed to be in the Lebesgue space $\mathcal{X}_{A}^{2}$, see Section 4 where $A$ is an $H$-algebra. For the algebra $A, A^{\infty}$ is supposed to be dense in $A$. It can be proved, see $[9,10]$, that $Y$-periodic functions and separable subalgebras of almost periodic functions mentioned in Section 3 satisfy the assumptions.

\section{Local and homogenized problem}

Further procedure is analogous to the one in the periodic problem. According to the equation, the following local bilinear form

$$
\widehat{a}(u, v)=\sum_{i, j=1}^{N} \int_{\Delta(A)} \mathcal{G}\left(a_{i j}\right)(s) \frac{\partial u}{\partial s_{j}} \frac{\partial v}{\partial s_{i}} \mathrm{~d} \beta(s)
$$

is introduced. As in the periodic case, let $\chi^{j} \in H^{1}(\Delta(A)) / \mathbb{R}, j=1, \ldots, N$ be the solution to the local problem: Find $\chi^{j} \in H^{1}(\Delta(A)) / \mathbb{R}$ such that

$$
\widehat{a}\left(\chi^{j}-s_{j}, v\right)=0 \quad \forall v \in H^{1}(\Delta(A)) / \mathbb{R} .
$$

Then, the homogenized coefficients $b_{i j}$ are given by

$$
b_{i j}=\int_{\Delta(A)}\left[\widehat{\mathcal{G}}\left(a_{i j}\right)-\sum_{k=1}^{N} \int_{\Delta(A)} \mathcal{G}\left(a_{i k}\right) \frac{\partial \chi^{j}}{\partial s_{k}}(x, s)\right] \mathrm{d} \beta(s) .
$$

Let $u^{*}$ be the solution to the homogenized problem, i.e., the problem (5.1) with the coefficients $a_{i j}^{\varepsilon}$ replaced by the homogenized coefficients $b_{i j}$ :

$$
-\sum_{i, j=1}^{N} \frac{\partial}{\partial x_{i}}\left(b_{i j} \frac{\partial u^{*}}{\partial x_{j}}\right) \equiv-\sum_{i, j=1}^{N} b_{i j} \frac{\partial^{2} u^{*}}{\partial x_{j} \partial x_{i}}=f \text { in } \Omega, \quad u^{*}=0 \quad \text { on } \partial \Omega .
$$

\section{Homogenization result}

The homogenization result can be summed up in the following theorem:

Theorem 5.1. Under the hypothesis mentioned above, the sequence of solutions $u^{\varepsilon}$ to problems (5.1) converges to the solution $u^{*}$ of the homogenized problem (5.5):

$$
u^{\varepsilon} \rightarrow u^{*} \text { weakly in } H_{0}^{1}(\Omega) \text { and strongly in } L^{2}(\Omega) \text {. }
$$

\section{Concluding Remarks}

Although, in the simplest case, the outlined approach is formally rather complicated, the idea of a spectrum of Banach algebra is fruitful, see $[8,9,13,11]$. The spectrum $\Delta(A)$ of the algebra $A$ with mean value operator $M$ replaces the period $Y$ of the $Y$-periodic coefficients in the periodic homogenization, the formulas (5.2), (5.3), (5.4) are analogous to those of the periodic homogenization.

The approach by Nguetseng in [9], [10] deals with complex Banach algebras, for homogenization of problems with real valued coefficients, the real Banach spaces and algebras seem to be sufficient. Thus, in the real case, the theory can be simplified, e.g., the condition for complex conjugate: "If $u \in A$, then also the complex conjugate $\bar{u} \in A$ " can be omitted.

This general approach was further developed and adopted for parabolic problems, perforated domains, reiterated homogenization, etc., see, e.g., [13, 7] and references therein. 


\section{REFERENCES}

[1] G. Allaire, Homogenization and two-scale convergence. SIAM J. Math. Anal. 23 (1992), 1482-1518.

[2] A. Bensoussans, J. L. Lions and G. Papanicolaou, Asymptotic Analysis for Periodic Structers, North Holland 1978.

[3] D. Cioranescu, A. Damlamian and G. Griso, The periodic unfolding method in homogenization, SIAM J. Math. Anal. 40 (2008), 1585-1620.

[4] J. Franců, Modification of unfolding approach to two-scale convergence, Mathematica Bohemica 135 (2010), 403-412.

[5] J. Franců and N. Svanstedt, Some remarks on two-scale convergence and periodic unfolding, Appl. Math. 57 (2012), 359-375.

[6] R. Larsen, Banach Algebras, Marcel Dekker, New York, 1973.

[7] D. Lukkassen, G. Nguetseng, H. Nnang and P. Wall, Reiterated homogenization of nonlinear monotone operators in a general deterministic setting, J. Funct. Spaces Appl. 7 (2009), 121152.

[8] G. Nguetseng, A general convergence result for a functional related to the theory of homogenization, SIAM J. Math. Anal. 20 (1989), 608-623.

[9] G. Nguetseng, Homogenization structures and applications I, Z. Anal. Anwen. 22 (2003), 73-107.

[10] G. Nguetseng, Homogenization structures and applications II, Z. Anal. Anwen. 23 (2004), 482-508.

[11] G. Nguetseng and N. Svanstedt, E-convergence, Banach J. Math. Anal. 5 (2011), 101-135.

[12] N. Svanstedt, Stochastic homogenization of a class of monotone eigenvalue problems, Appl. Math. 55 (2010), 385-404.

[13] J. L. Woukeng, $\Sigma$-convergence of nonlinear monotone operators in perforated domains with holes of small size, Appl. Math. 54 (2009), 465-489.

[14] W. Żelazko, Banach Algebras, Elsevier, Amsterdam, 1973.

Jan Franců, Institute of Mathematics, Faculty of Mechanical Engineering, Brno University of Technology, Technická 2, 61669 Brno, Czech Republic

e-mail: francu@fme.vutbr.cz 